\title{
Fachkräftemangel in Ost- und Westdeutschland? Eine betriebliche Perspektive
}

Das Thema „Fachkräftemangel“ steht derzeit im Fokus der öffentlichen Aufmerksamkeit und wird sehr kontrovers diskutiert. Die aktuell (noch) gute Lage auf dem Arbeitsmarkt spiegelt sich vor allem in einer hohen betrieblichen Arbeitskräftenachfrage wider. Daraus allein lässt sich jedoch noch keine Quantifizierung eines „Fachkräftemangels“ ableiten. Jedoch können verschiedene Kennzahlen - wie das Verhältnis von Arbeitslosen und offenen Stellen oder Stellenbesetzungsdauern - dazu beitragen, die Situation in unterschiedlichen Wirtschaftsbereichen zu beleuchten. Ergänzend haben wir Betriebe in Ost- und Westdeutschland befragt, inwieweit sie sich vom Fachkräftemangel betroffen fühlen.

MARTIN DIETZ, ALEXANDER KUBIS, ANNE MÜLLER

\section{Einleitung}

Die Kombination aus Fachkräftemangel und persistenter Arbeitslosigkeit ist eines der negativen Zukunftsszenarien für den Arbeitsmarkt. Mit Blick auf das schrumpfende Erwerbspersonenpotenzial, die bereits hohen und tendenziell eher steigenden Qualifikationsanforderungen der Betriebe sowie angesichts des noch immer zu großen Anteils von Menschen ohne Schul- oder Berufsabschluss erscheint dieses Szenario als nicht unrealistisch.

Wo stehen wir heute? Betrachtet man die aktuelle Situation am Arbeitsmarkt, ist zunächst unbestritten, dass verfestigte Arbeitslosigkeit vor allem für Personen mit geringer formaler Qualifikation ein andauerndes Problem ist. Dies gilt, obwohl sich die Beschäftigungssituation in den vergangenen Jahren deutlich verbessert hat und von einer gestiegenen Matching-Effizienz am Arbeitsmarkt auch Arbeitslose im Grundsicherungssystem des Sozialgesetzbuch Zweites Buch (SGB II) profitiert haben (Klinger/Rothe 2012).

Über die Frage, ob in Deutschland bereits ein Fachkräftemangel zu beobachten ist, und wenn ja, auf welche Arbeitsmarktsegmente er sich bezieht, gibt es dagegen kontroverse Meinungen (Brenke 2011; Koppel 2011). Die Diskussion an sich zeigt jedoch, dass sich die Situation auf vielen Teilarbeitsmärkten zu drehen beginnt. Wo Betriebe es lange Zeit gewohnt waren, aus einem Pool geeigneter Bewerber wählen zu können, zeichnet sich nun eine gewisse Knappheit ab. Diese veränderte Marktsituation dürfte dazu beitragen, dass Betriebe Stellenbesetzungen vermehrt als schwierig empfinden und die Debatte um fehlende Fachkräfte an Fahrt gewinnt.

Dieser Beitrag zeichnet ein differenziertes Bild der betrieblichen Arbeitskräftenachfrage. Als Basis hierfür dient die vom Institut für Arbeitsmarkt- und Berufsforschung (IAB) durchgeführte Erhebung des Gesamtwirtschaftlichen Stellenangebots (EGS). ${ }^{\ominus}$ Diese repräsentative Betriebsbefragung eröffnet im Zusammenspiel mit anderen Arbeitsmarktdaten einen nach Ost- und Westdeutschland differenzierten Blick auf Probleme bei der Fachkräfterekrutierung und zeigt zudem, in welchem Ausmaß sich Betriebe innerhalb dieser Regionen bereits durch geeignete Gegenmaßnahmen auf eine sich wandelnde Marktsituation eingestellt haben.

Die Erhebung wurde vom IAB in Zusammenarbeit mit Economix Research \& Consulting konzipiert und durchgeführt. Aktuelle Ergebnisse und weiterführende Informationen vgl. unter: www.iab.de/stellenerhebung. 


\section{Die betriebliche Arbeitskräfte- nachfrage}

\subsection{Gesamtwirtschaftliche Trends}

Die gesamtwirtschaftliche Arbeitskräftenachfrage lässt sich im Rahmen der EGS bestimmen. Im 4. Quartal 2011 liegt sie mit 1.130.000 offenen Stellen wieder auf dem Niveau vor Beginn der Finanz- und Wirtschaftskrise. Dabei werden nur Stellen auf dem ersten Arbeitsmarkt betrachtet, also keine staatlich geförderten Stellen wie Arbeitsgelegenheiten oder Arbeitsbeschaffungsmaßnahmen. Gut 850.000 Stellen sind sofort zu besetzen und bilden damit den akuten Bedarf der Betriebe ab. Die unterschiedliche Wirtschaftskraft in Ost- und Westdeutschland spiegelt sich auch in der Zahl der offenen Stellen wider: In den alten Bundesländern (ohne Berlin) sind rund 960.000 offene Stellen zu verzeichnen (davon gut 730.000 sofort zu besetzen), in Ostdeutschland sind es 170.000 (120.000).

Die Vakanzrate zeigt an, wie hoch der Anteil der noch ungedeckten Nachfrage in Form der sofort zu besetzenden Stellen an der gesamten Arbeitskräftenachfrage (als Summe aus sofort zu besetzenden Stellen und sozialversicherungspflichtiger Beschäftigung) ist. Die Vakanzrate ist im Zuge der wirtschaftlichen Erholung nach der Finanz- und Wirtschaftskrise wieder angestiegen, hat sich seitdem stabilisiert und lag Ende 2011 bei einem Wert von 2,9 \% (Abbildung 1). Knapp 3 \% der gesamten Arbeitskräftenachfrage sind also noch unbefriedigt. Obwohl die Entwicklungen zwischen den Landesteilen dieselbe Tendenz aufweisen, werden Niveau-Unterschiede zwischen Ost- und Westdeutschland sichtbar: Die ungedeckte Arbeitsnachfrage nimmt in den alten Bundesländern einen größeren Anteil am Beschäftigungspotenzial ein, was als Zeichen für einen nachfragestärkeren Arbeitsmarkt angesehen werden kann.

Trotz einer gewissen Annäherung lassen sich die stärkeren Probleme des ostdeutschen Arbeitsmarktes auch an der vergleichsweise höheren Arbeitslosenquote ablesen ( $A b$ bildung 1). Trotzdem bestätigt auch ein Blick auf die Entwicklung der Arbeitslosigkeit den positiven Trend am deutschen Arbeitsmarkt.

Die aktuelle Fachkräftedebatte verläuft also vor dem Hintergrund einer sich verbessernden Arbeitsmarktlage, für die sowohl strukturelle als auch konjunkturelle Ursachen verantwortlich gemacht werden können (Gartner/Klinger 2007, S. 619). Der Blick auf die aggregierten Daten spricht zunächst dafür, dass sich die Situation in den alten Bundesländern durch eine höhere Arbeitsmarktanspannung auszeichnet, dass also eine im Vergleich der Regionen höhere Vakanzrate (Arbeitsnachfrage) auf eine geringere Arbeitslosenquote (Arbeitsangebot) trifft. In Ostdeutschland dagegen ist die Arbeitslosenquote transformationsbedingt deutlich höher und steht einem nicht nur absolut, sondern auch relativ betrachtet geringeren Stellenangebot gegenüber. Hier förderte über lange Zeit das im Vergleich zur Produk- tivität überdurchschnittlich hohe Lohnniveau eher kapitalintensive und tendenziell personalfreisetzende (Ersatz-) Investitionen (Ragnitz et al. 2004, S. 32).

\subsection{Regionale und berufsspezifische Unterschiede}

Ein differenzierter Blick auf die einzelnen Bundesländer bestätigt die strukturellen Unterschiede zwischen dem ostdeutschen und westdeutschen Arbeitsmarkt und offenbart ein sehr heterogenes Bild über alle Bundesländer hinweg (Abbildung 2). ${ }^{2}$ Der Schnittpunkt zwischen $\mathrm{x}$ - und $\mathrm{y}$-Achse in Abbildung 2 stellt den bundesdeutschen Durchschnitt im 4. Quartal 2010 dar, die Diagonale die hierzu äquidistanten Zustände. Dies bedeutet, dass sich alle Bundesländer links der Diagonale im interregionalen Vergleich durch eine geringere Arbeitslosenquote und/oder eine höhere Anzahl an sofort zu besetzenden Stellen auszeichnen. Im oberen linken Quadranten finden sich Bundesländer mit einer Kombination aus einer unterdurchschnittlichen Arbeitslosenquote und einer überdurchschnittlichen Vakanzrate. In den westdeutschen Flächenländern Bayern, Baden-Württemberg und Hessen dürften also am ehesten Rekrutierungsprobleme zu erwarten sein. Dagegen verorten sich im unteren rechten Quadranten Bundesländer mit einer vergleichsweise hohen Arbeitslosigkeit und einem relativ geringen Potenzial an neuen Jobs. Hier befinden sich vor allem die ostdeutschen Bundesländer.

Bislang wurden Arbeitskräftenachfrage und Arbeitslosigkeit nebeneinander ausgewiesen. Führt man sie zusammen und bildet das Verhältnis zwischen Arbeitslosen und offenen Stellen, so erhält man einen Indikator für die Knappheit am Arbeitsmarkt. Dabei ist zunächst zu beachten, dass diese Relation stark durch konjunkturelle Einflüsse geprägt ist. In Aufschwüngen zieht die betriebliche Nachfrage nach Arbeitskräften an, die Zahl der offenen Stellen steigt und die Arbeitslosigkeit geht zurück - die umgekehrte Entwicklung zeigt sich in Rezessionen und führt zu einem Anstieg der Relation. Dies galt auch in der zurückliegenden Finanzkrise - obwohl Betriebe in einem zuvor noch nicht gekanntem Ausmaß Arbeitskräfte gehalten haben (Dietz et al. 2011). Während dadurch die Arbeitslosigkeit gemessen an dem starken Einbruch des Bruttoinlandsprodukts (BIP) überraschend stabil blieb, reduzierte sich die Zahl der offenen Stellen deutlich. Nach Ende der Wirtschaftskrise sanken die Arbeitslosenzahlen wieder und die Zahl der offenen Stellen näherte sich dem wirtschaftsstarken Vorkrisenniveau. Im 2. Quartal 2011 lag der Quotient bei einem Wert von 4,2, d. h. auf 100 sofort zu besetzende Stellen kamen in

(2) Erst in jüngster Vergangenheit führten der demografische Wandel und eine zurückhaltende Lohnentwicklung zu einem - von einer hohen Arbeitslosenquote ausgehenden sinkenden Arbeitskräfteangebot bei gleichzeitig steigender Rentabilität arbeitsintensiverer Investitionen. 


\section{Entwicklung von Vakanzrate ${ }^{1}$ und Arbeitslosenquote ${ }^{2}$, 1. Quartal 2006 - 4. Quartal 2011}
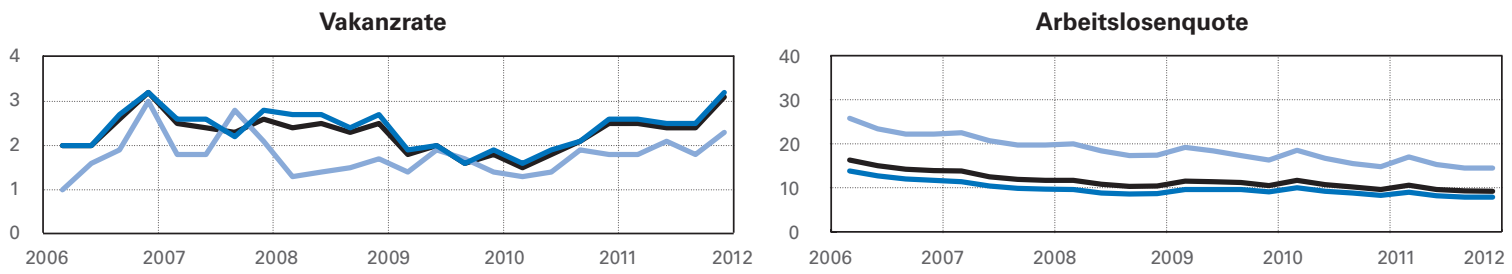

Deutschland durchschnittlich 420 arbeitslos gemeldete Personen.

Die entsprechende Knappheitsrelation lässt sich für das 4. Quartal 2010 auch für Berufssegmente ausweisen (Abbildung 3). Ein Blick darauf ist sinnvoll, weil der deutsche Arbeitsmarkt stark beruflich strukturiert ist. Die betrieblichen Anforderungen bei einer Rekrutierung weisen in der Regel eine klare berufliche Richtung aus, sodass die Passung zwischen dem beruflichen Profil der Arbeitslosen und den Wünschen der Arbeitgeber eine besondere Bedeutung hat.

Die Betrachtung nach Berufsfeldern zeigt, dass es einige Bereiche gibt, die ein unterdurchschnittliches Verhältnis von Arbeitslosen $\mathrm{zu}$ sofort $\mathrm{zu}$ besetzenden Stellen aufweisen. ${ }^{3}$ Besonders eng erscheinen die beruflichen Teilarbeitsmärkte bei den Gesundheitsdiensten, im Elektrobereich sowie bei der Gruppe der Ingenieure, Chemiker, Physiker und Mathematiker. In diesen Segmenten mit Werten unter 1 steht nicht jeder offenen Stelle auch ein Arbeitsloser gegenüber. Auf der anderen Seite finden sich Berufssegmente, in denen ein starker Arbeitskräfteüberschuss zu beobachten ist - in den Bereichen Ordnung und Sicherheit, sonstige Dienstleistungen sowie Hoch- und Tiefbau kommen 20 und mehr Arbeitslose auf eine sofort zu besetzende Stelle.

Einschränkend muss jedoch beachtet werden, dass die hier abgetragenen Berufsfelder eine Vielzahl an heterogenen Berufen vereinen und sich somit neben räumlichen auch qualifikatorische Unschärfen ergeben. Außerdem betrachtet man mit den Arbeitslosen auf Seite des Arbeitsangebots nur ein Teilsegment und vernachlässigt damit, dass ein gewisser Teil der Nachfrage durch erwerbstätige Jobwechsler und Absolventen vom Ausbildungsmarkt gedeckt werden kann. Allerdings erfordert eine (hochspezialisierte) Tätigkeit wie beispielsweise im Falle von Berufen aus den Bereichen Mathematik, Informatik, Naturwissenschaft und Technik (MINT) eine Qualifikation, deren Erwerb langwierig
ABB. 2

Vakanzrate $^{1}$ und Arbeitslosenquote ${ }^{2}$ am 1. Arbeitsmarkt nach Bundesländern, 4. Quartal 2010

Angaben in Prozent

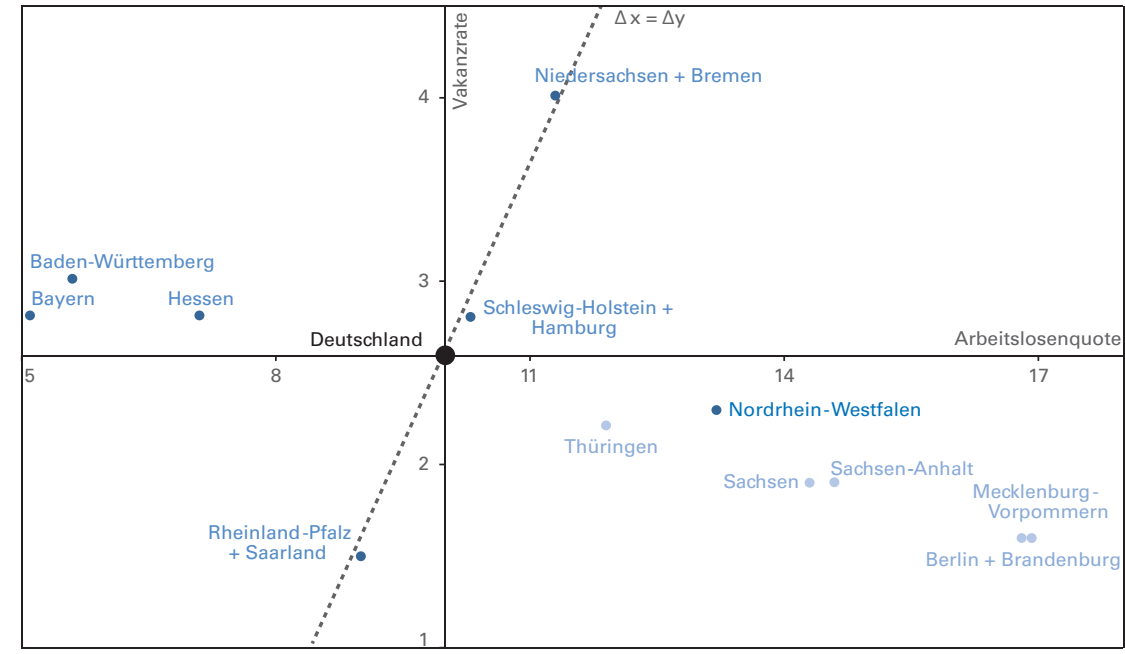

Quelle: IAB-Erhebung des Gesamtwirtschaftlichen Stellenangebots; Statistik der BA. 
ABB. 3

\section{Arbeitslose pro sofort zu besetzender Stelle nach Berufsfeldern ${ }^{1}$, 4. Quartal 2010}

Angaben in absoluten Zahlen

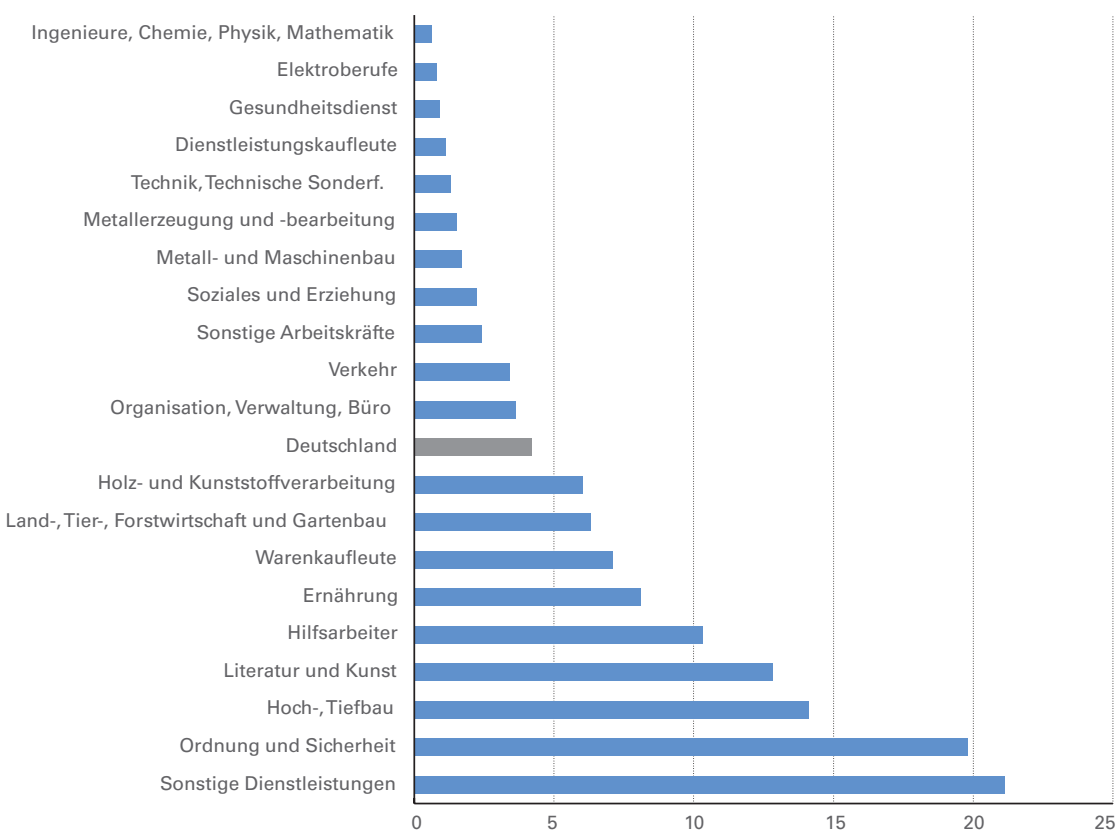

1) ausgewählte Berufsfelder der KIdB 92 (Klassifizierung der Berufe, Version Statistisches Bundesamt 1992).

und schwierig ist. So könnten diese Fachkräfte trotz geeigneter Signale dennoch nur mittel- bis langfristig neu ausgebildet und akquiriert werden, insbesondere, da die Substitutionsfähigkeit durch artverwandte Berufe vergleichsweise gering ist. Das Finden eines neuen Marktgleichgewichts benötigt hier entsprechend viel Zeit.
Etwas anders sieht es im Bereich der Gesundheitsdienste mit seiner Vielzahl an Pflegeberufen aus. Sowohl die größere Zahl des bestehenden Fachkräfteangebots als auch die stärkere fachliche Nähe zwischen den einzelnen Berufen eröffnen grundsätzlich mehr Spielräume, um mögliche Engpässe aufzufangen. Dafür besteht bei Pflegeberufan an anderer Stelle eine Restriktion, was die Anreize zur Erhöhung des Arbeitsangebotes in diesem Segment angeht: Der Lohnmechanismus ist als treibendes Marktkorrektiv nur schwach ausgeprägt, da öffentliche Einnahmerigiditäten den Ausgabespielraum (Lohn) quasi deckeln. Dies gilt im Bereich des Gesundheitsdienstes, der trotz der Zunahme privater Anbieter noch immer überdurchschnittlich stark öffentlich finanziert ist.

\section{Ergebnisse der Betriebsbefragung}

\subsection{Fachkräftemangel aus betrieblicher Sicht}

Betrachtet man also die allgemeinen Rahmenbedingungen am Arbeitsmarkt sowie erste Indikatoren für die Passung zwischen Angebot und Nachfrage, so zeigt sich kein eindeutiges Bild. Während es in bestimmten Arbeitsmarktsegmenten kaum vorstellbar ist, dass Betriebe Rekrutierungsprobleme haben, scheint dies in anderen Bereichen durchaus möglich.

Um eine genauere Vorstellung der erlebten Rekrutierungsprobleme in Betrieben zu erhalten, wurden diese im 4. Quartal 2010 explizit gefragt, ob sie sich derzeit vom Fachkräftemangel betroffen fühlen. 16,7 \% der rund 15.000 teilnehmenden Betriebs- und Verwaltungsstätten antworteten in diesem Sinne. Tabelle 1 stellt die Ergebnisse getrennt nach Ost- und Westdeutschland sowie nach drei Betriebsgrößenklassen dar. Dabei zeigt sich, dass Betriebe in Westdeutsch-

TABELLE 1

Betriebe mit aktiver Personalsuche* und vom Fachkräftemangel betroffene Betriebe**, 4. Quartal 2010 Anteile in Prozent

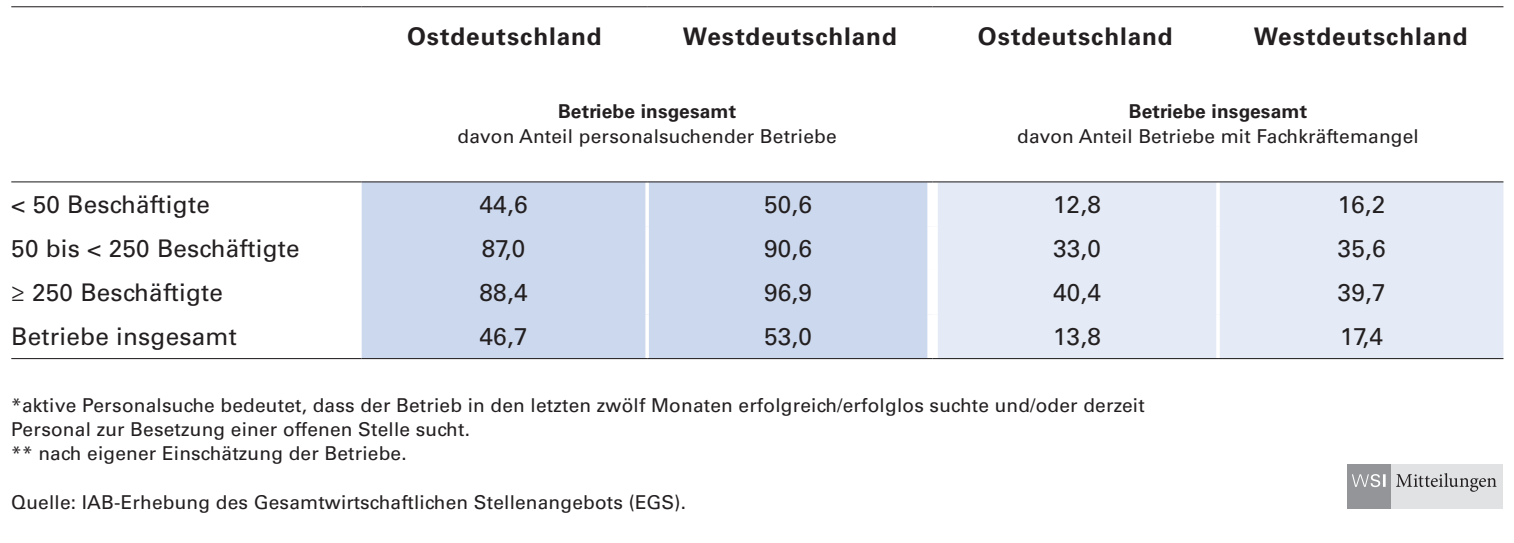


Schnittmenge von Betrieben mit aktiver Personalsuche* und Betrieben mit Fachkräftemangel**, 4. Quartal 2010

Anteile in Prozent

\begin{tabular}{|c|c|c|c|c|}
\hline & Ostdeutschland & Westdeutschland & Ostdeutschland & Westdeutschland \\
\hline & \multicolumn{2}{|c|}{$\begin{array}{c}\text { Betriebe mit Fachkräftemangel } \\
\text { davon Anteil personalsuchender Betriebe }\end{array}$} & \multicolumn{2}{|c|}{$\begin{array}{l}\text { Personal suchende Betriebe } \\
\text { davon Anteil Betriebe mit Fachkräftemangel }\end{array}$} \\
\hline$<50$ Beschäftigte & 91,0 & 88,9 & 26,1 & 28,4 \\
\hline 50 bis $<250$ Beschäftigte & 97,1 & 98,4 & 36,9 & 38,7 \\
\hline$\geq 250$ Beschäftigte & 96,3 & 99,5 & 44,1 & 40,7 \\
\hline Betriebe insgesamt & 91,7 & 90,1 & 27,2 & 29,5 \\
\hline
\end{tabular}

*aktive Personalsuche bedeutet, dass der Betrieb in den letzten zwölf Monaten erfolgreich/erfolglos suchte und/oder derzeit Personal zur Besetzung einer offenen Stelle sucht.

*** nach eigener Einschätzung der Betriebe.

Quelle: IAB-Erhebung des Gesamtwirtschaftlichen Stellenangebots (EGS).

land mit 17,4 \% etwas häufiger einen Fachkräftemangel beklagen als in Ostdeutschland (13,8 \%). Das Problem scheint zudem eher bei mittleren und größeren Betrieben aufzutreten. Dies kann allerdings auch damit zusammenhängen, dass diese Betriebe im selben Zeitraum häufiger Personal rekrutieren. Damit steigt auch die Wahrscheinlichkeit, dass bei einer der zahlreicheren Stellenbesetzungen Probleme auftreten, die dann als Fachkräftemangel wahrgenommen werden. Der Anteil der Betriebe mit aktiver Personalsuche findet sich ebenfalls in Tabelle 1. Mit 51,7 \% suchten etwas mehr als die Hälfte der Betriebe insgesamt im Jahr 2010 Personal. Westdeutsche Betriebsstätten suchten im beobachteten Zeitraum häufiger Personal und gaben an, verstärkt vom Fachkräftemangel betroffen zu sein. Dies scheint im Lichte der im vorherigen Abschnitt aufgeführten allgemeinen Arbeitsmarktdaten plausibel (Abbildung 1).

Beschränkt man die Auswertung der Frage nach einem Fachkräftemangel auf Betriebe, die im relevanten Zeitraum auch aktiv Personal suchten, so sieht knapp jeder dritte Betrieb einen Fachkräftemangel (Tabelle 2). Mit Blick auf die Ost-West-Dimension bestätigen sich die grundsätzlichen Ergebnisse aus Tabelle 1. Dies gilt auch für die Betriebsgrößenklassen, wobei sich hier ein deutlicher Anstieg der Werte bei der untersten Größenklasse zeigt, was darauf zurückzuführen sein dürfte, dass die Differenz zwischen der Gesamtzahl der Betriebe in dieser Größenklasse und der Personal suchenden Betriebe besonders hoch ist. Umgekehrt wird deutlich, dass knapp $10 \%$ der Betriebe, die sich vom Fachkräftemangel betroffen fühlen, im Jahr 2010 kein Personal suchten. Dieser zunächst irritierende Befund lässt sich neben einer diffusen Angst vor einer sich ändernden Arbeitsmarktsituation auch damit erklären, dass Fachkräftemangel nicht nur durch ein Fehlen von geeigneten Bewerbern gekennzeichnet sein kann, sondern auch dadurch, dass die bestehende Belegschaft eines Betriebes die notwendigen fachlichen Kenntnisse und Fertigkeiten nicht (mehr) aufweist, beispielsweise, weil interne Qualifizierungsund Weiterbildungsmaßnahmen ausgeblieben sind.

Letztlich bestätigt sich aber auch bei einer direkten Befragung der Betriebe zu Fachkräfteproblemen, dass sowohl in West- als auch in Ostdeutschland ein Teil der Betriebsstätten zumindest temporär einem Arbeitskräfteangebot gegenübersteht, das vom Anforderungsprofil ihrer zu besetzenden Stelle abweicht. Hinzu kommt der allgemeine Befund, dass Knappheiten vor allem in prosperierenden Wirtschaftsräumen auftreten (Abbildung 2).

Die Betriebe nennen verschiedene Ursachen, die aus ihrer Sicht den erlebten Fachkräftemangel begründen ( $A b$ bildung 4). So sehen rund $60 \%$ der Betriebe den Hauptgrund für ihre Betroffenheit vom Fachkräftemangel darin, dass schlichtweg zu wenige Fachkräfte auf dem Arbeitsmarkt verfügbar sind. Ebenfalls $60 \%$ geben an, dass Bewerber für die (hochkomplexe) Tätigkeit nicht ausrei-

ABB. 4

Indikatoren für einen Fachkräftemangel aus betrieblicher Sicht, 4. Quartal 2010 Angaben in Prozent

Ostdeutschland

Westdeutschland

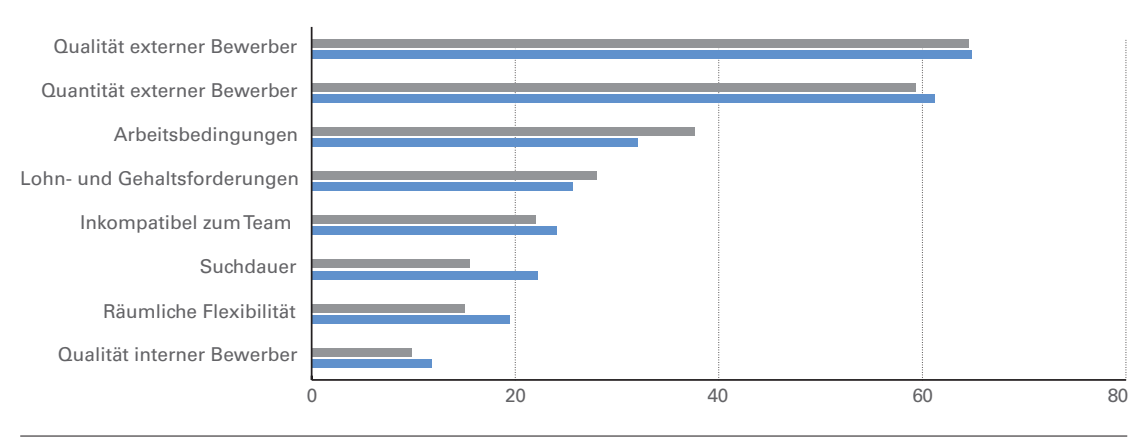

Quelle: IAB-Erhebung des Gesamtwirtschaftlichen Stellenangebots. 
ABB.

\section{Kompetenzmängel externer Bewerber aus Sicht der Betriebe' ', 4. Quartal 2010 \\ Angaben in Prozent \\ Ostdeutschland \\ Westdeutschland}

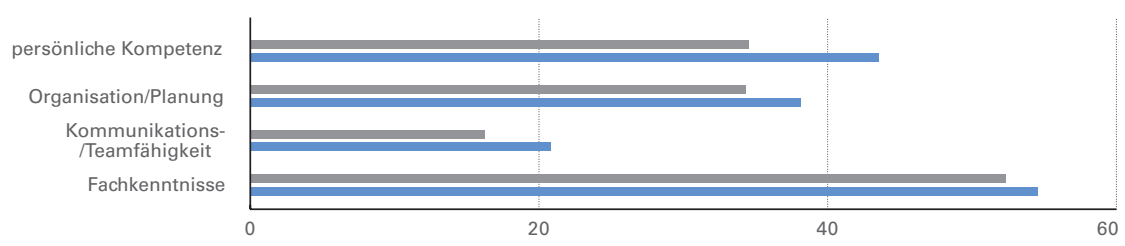

1) Die Fragestellung bei Betrieben mit Fachkräftemangel wegen kompetenzschwacher Bewerber lautete: Wie häufig stellten Sie welchen Mangel an Kompetenzen bei den externen Bewerbern/Bewerberinnn fest? (Mehrfachnennungen möglich).

Quelle: IAB-Erhebung des Gesamtwirtschaftlichen Stellenangebots.

\section{ABB. 6}

\section{Handlungsfelder zur Qualifizierung der internen Belegschaft ${ }^{1}$, 4. Quartal 2010}
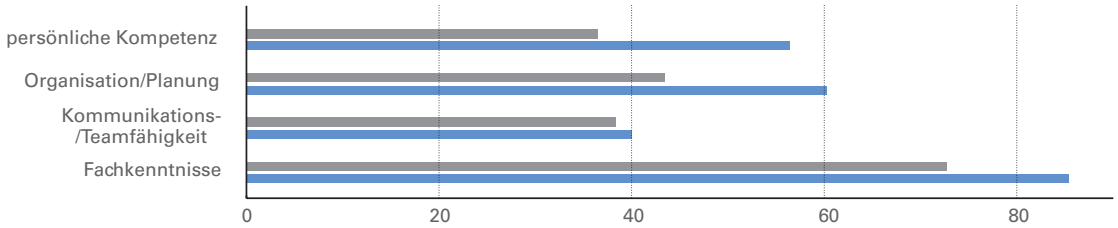

Die Fragestellung bei Betrieben mit Fachkräften und Qualifikationsmängeln der internen Belegschaft lautete: Wie hoch schätzen Sie den Handlungsbedarf zur betrieblichen Leistungserhaltung/-erhöhung bzgl. der Kompetenzen der eigenen Belegschaft ein? (Mehrfachnennungen möglich)

Quelle: IAB-Erhebung des Gesamtwirtschaftlichen Stellenangebots.

chend qualifiziert sind. Mit erkennbarem Abstand folgen voneinander abweichende Lohn- und Arbeitsvorstellungen bis hin zu fehlender räumlicher Flexibilität. Neben diesen eher externen Ursachen gibt Abbildung 4 auch einen ersten Hinweis auf ein zusätzliches, eher „weiches“ Problem: Sowohl in Ost- als auch in Westdeutschland geben etwa $20 \%$ der Betriebe an, dass potenzielle Bewerber nicht in das bestehende Team passen.

Auch interne Gründe können, wie bereits erwähnt, Fachkräftemangel bedingen: dann nämlich, wenn die bestehende Belegschaft nicht (mehr) ausreichend qualifiziert ist und dadurch die Produktivität und Leistungsfähigkeit des Betriebes beeinträchtigt wird. Aus Abbildung 4 wird jedoch ersichtlich, dass mangelnde Kompetenzen der externen Bewerber von den Betrieben wesentlich häufiger als Ursache für Fachkräftemangel genannt werden als eine unzureichende Qualifikation der bestehenden Belegschaft.

In einem weiteren Fragebogenabschnitt wurde nun zum einen danach gefragt, welche Kompetenzen der Bewerber als nicht ausreichend eingeschätzt werden (Abbildung 5), bzw. wo Handlungsbedarf zum Erhalt bzw. zur Verbesserung der Leistungsfähigkeit der internen Belegschaft besteht (Abbildung 6). Dabei wird die Auswertung jeweils auf diejenigen Betriebe beschränkt, die von einem Fachkräftemangel aufgrund mangelnder Kompetenzen der externen Bewerber bzw. ihrer bestehenden Belegschaft berichteten.

Wenn Betriebe Bewerber als unzureichend qualifiziert einschätzen, so liegt dies in der Hälfte der Fälle in einem Mangel an fachlichen Kompetenzen begründet. Auch das Fehlen von persönlichen Kompetenzen, wie Zuverlässigkeit und Leistungsbereitschaft, ist ein häufig genannter Grund für „,externen“ Fachkräftemangel. Seltener scheint dieser auf mangelnder Kommunikations- und Teamfähigkeit zu beruhen. Dieser Befund gilt sowohl für West- wie auch für Ostdeutschland, wobei über alle Kompetenz- und Handlungsfelder hinweg westdeutsche Betriebe eine größere Anzahl von Gründen angeben. Es ist aber auch ersichtlich, dass sich bestehende Strukturunterschiede zwischen den Regionen im „Empfinden“ der Betriebe nur marginal widerspiegeln. Dies lässt darauf schließen, dass die Kompetenzen der auf dem Arbeitsmarkt verfügbaren (und mobilen) Fachkräfte im Osten nicht grundlegend von denen im Westen abweichen.

Aus Sicht der Betriebe, die angegeben haben, von einem internen Fachkräfteproblem betroffen zu sein, deren Problem also (auch) auf eine nicht (mehr) ausreichend qualifizierte Belegschaft zurückzuführen ist, liegt ein wichtiger Anknüpfungspunkt in der Verbesserung der fachlichen Qualifikation ihrer Mitarbeiter (Abbildung 6). Weit über $80 \%$ der betroffenen Betriebe sehen hier Handlungsbedarf. An zweiter Stelle folgt die Einschätzung, dass die organisatorischen Fähigkeiten der Mitarbeiter (Planung, Führung, Kontrolle) verbesserungsbedürftig seien. In Bezug auf Qualifikationsmerkmale, die eher als Soft Skills eingestuft werden, zeigen sich zum Teil Unterschiede in der Einschätzung west- bzw. ostdeutscher Betriebe: Fast gleichrangig bewerten Ost- und Westdeutsche Betriebe die Kommunikationsund Teamfähigkeit ihrer Belegschaft als Handlungsfeld. Mit Blick auf die Verbesserung persönlicher Kompetenzen wird allerdings im Westen deutlich höherer Handlungsbedarf gesehen als im Osten Deutschlands. Ein Grund hierfür dürfte das lange Zeit vorherrschende Überangebot qualifizierter Fachkräfte im Osten Deutschlands sein, die über die geforderte Berufserfahrung und damit auch entsprechend eingeübte „weiche“ Qualifikationen verfügten.

\subsection{Betriebliche Reaktionen auf Fachkräfteengpässe}

In ausgewählten Regionen und Berufssegmenten gibt es also Anzeichen, dass Betriebs- und Verwaltungsstätten einem verknappten Fachkräfteangebot gegenüberstehen. Wie reagieren Betriebe nun auf solche Rekrutierungsschwierigkeiten?

Stellen Betriebe im Verlauf des Rekrutierungsprozesses fest, dass es schwierig ist, einen geeigneten Bewerber zu 
ABB. 7

\section{Kompromisse der Betriebe bei Neuein-} stellungen ${ }^{1}$, 4. Quartal 2006-2010

Angaben in Prozent

Lohn

Qualifikation

Erfahrung

Westdeutschland

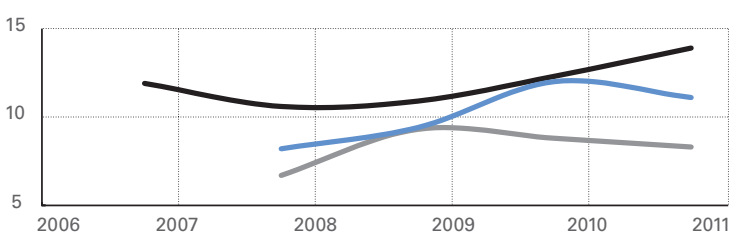

Ostdeutschland

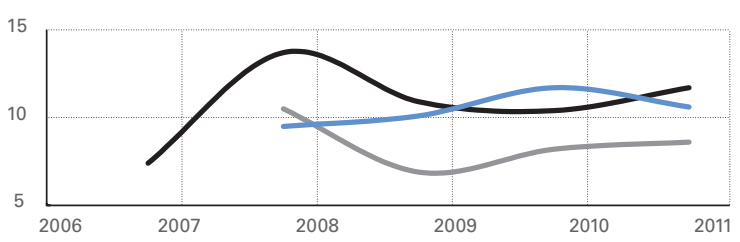

1) Die Fragestellung bezogen auf den letzten Fall einer erfolgreichen, sozialversicherungspflichtigen Neueinstellung lautete: War es für die Besetzung der Stelle erforderlich, mehr Lohn als vorgesehen zu zahlen?

Unterscheidet sich der Mitarbeiter/die Mitarbeiterin im

Hinblick auf Qualifikation/Berufserfahrung [ ] von dem,

was Sie ursprünglich gesucht haben?

Quelle: IAB-EGS (1989-2012).

finden, so muss dies nicht unbedingt in einem Einstellungsverzicht enden. Um den Abbruch der Personalsuche zu vermeiden, kann der Arbeitgeber bei unterschiedlichen Eigenschaften des Stellenprofils Kompromisse eingehen. Abbildung 7 bildet die Fälle einer erfolgreichen Neueinstellung ab, bei denen Betriebe Kompromisse in Hinblick auf die Entlohnung, Qualifikation oder Berufserfahrung eingegangen sind.
In Westdeutschland ist seit einigen Jahren ein Anstieg beim Lohnkompromiss zu beobachten, wobei aktuell bei knapp 14 \% aller Neueinstellungen ein höherer Lohn gezahlt wird als ursprünglich vom Betrieb vorgesehen. Dies spricht für eine Fachkräfteverknappung, denn es gelingt in diesen Fällen wohl nur durch höhere Lohnzahlungen, entsprechend qualifiziertes Personal zu akquirieren. Etwas seltener werden Kompromisse bei der Berufserfahrung sowie bei der Qualifikation eingegangen. Hier sind keine großen Unterschiede zwischen Ost- und Westdeutschland festzustellen.

Dies gilt für Besetzungsprozesse, die schließlich doch zum Erfolg geführt haben. Das ist aber nicht immer so. Im schlechtesten Fall müssen Betriebe ihre Personalsuche einstellen, wenn kein Bewerber mit dem gewünschten Profil gefunden werden kann. Dies war im Jahr 2010 rund 517.000-mal der Fall und ist der stärkste Befund für einen Fachkräftemangel. Auch hier nannten die Betriebe als wichtigste Gründe, dass die Bewerberzahl insgesamt zu gering bzw. die Personen beruflich nicht gut genug qualifiziert gewesen seien. ${ }^{\circledR}$ Doch es zeigt sich, dass Betriebe durchaus flexibel auf Rekrutierungsprobleme reagieren können ( $\mathrm{Ta}$ belle 3). So fangen viele Betriebe eine erfolglose Personalsuche durch Mehrarbeit der Belegschaft auf. Auch der temporäre Einsatz von Leiharbeitnehmern stellt eine Alternative dar, die im Osten stärker als im Westen in Anspruch genommen wird.

In gut einem Viertel der Fälle muss auf Aufträge verzichtet werden. Hier führt das Rekrutierungsproblem dann tatsächlich zu einem betriebswirtschaftlichen Schaden. Auf die volkswirtschaftliche Ebene schlägt dies in der Regel nicht durch, denn häufig stehen Mitbewerber bereit, um den entsprechenden Auftrag auszuführen. Auch werden tech-

(4) Die Betriebsbefragung stößt hier jedoch an ihre Grenzen, da eine Repräsentativität der Aussagen nur für Ost- und Westdeutschland insgesamt garantiert werden kann.

TABELLE 3

\section{Betriebliche Reaktion auf erfolglose Personalsuche*, 4. Quartal 2010}

Anteile in Prozent

Ostdeutschland

\begin{tabular}{|c|c|}
\hline 44,2 & 38,1 \\
36,0 & 34,6 \\
27,2 & 29,8 \\
26,5 & 23,3 \\
15,0 & 10,1 \\
11,2 & 8,2 \\
9,2 & 7,5 \\
\hline
\end{tabular}

Aufgaben von anderen Mitarbeitern ohne Überstd. erledigt

Aufgaben von anderen Mitarbeitern durch Überstd. erledigt

Auftragsverzicht

technische/organisatorische Änderungen vorgenommen

Leiharbeit in Anspruch genommen

durch veränderte Marktsituation kein Bedarf mehr

offene Stelle wurde intern besetzt

* Fragestellung: „Welche Maßnahmen haben Sie aufgrund der erfolglosen Personalsuche ergriffen?” Mehrfachnennungen möglich, Hochrechnung auf Betriebe mit mindestens einem Suchabbruch.

Quelle: IAB-Erhebung des Gesamtwirtschaftlichen Stellenangebots (EGS). 
nische und organisatorische Veränderungen realisiert, um die gescheiterte Personalrekrutierung zu kompensieren. Dabei gilt es zu beachten, dass der von den Betrieben beklagte Mangel an individuellen fachlichen Kompetenzen sowohl der Bewerber als auch der Belegschaft durch technisch/organisatorische Veränderungen verstärkt werden kann, denn diese sind in Deutschland oftmals mit steigenden Qualifikationsanforderungen verbunden. Um generell ein Auseinanderdriften zwischen dem betrieblichem Anforderungsprofil der Stellen und dem Qualifikationsniveau der Belegschaft sowie der Bewerber zu vermeiden, gilt es, eine Vielzahl von Möglichkeiten zu nutzen. Der strukturelle/technologische Wandel macht lebenslanges Lernen geradezu unverzichtbar. Das betriebliche Ziel, die Beschäftigungsfähigkeit der Mitarbeiter zu erhalten, kann sich deswegen nicht im Abschluss einer Berufsausbildung erschöpfen. Zwar erklärt das allgemein hohe Ausbildungsniveau in Deutschland einen Teil der im OECD-Vergleich geringeren hiesigen Weiterbildungsquote; der aus Sicht der Betriebe empfundene Qualifikationsmangel weist aber gleichwohl deutlich auf die Notwendigkeit dauerhafter Weiterqualifizierung hin.

\section{Fazit}

Im Rückblick zeigt sich, dass die Arbeitsmarktsituation über Jahre hinweg geprägt war durch starke transformationsbedingte Schrumpfungs- und Alterungstendenzen in Ostdeutschland. Aber auch im Westen Deutschlands führte der strukturelle Wandel in einzelnen Regionen, wie dem Ruhrgebiet, zu starken Veränderungen am Arbeitsmarkt. Durch den Wegfall prägender (Alt-)Industrien, das Entstehen neuer Industriefelder (erneuerbare Energien) oder das anhaltende Wachstum bereits bestehender exportstarker Branchen im Süden Deutschlands kam es in ausgewählten Branchen zu verstärkten regionalen Ungleichgewichten in Bezug auf den Faktor Arbeit. Es lassen sich demnach verschiedene Ursachen identifizieren, die das nach wie vor sehr heterogene Bild eines auf einzelne Regionen sowie Branchen konzentrierten Fachkräfteengpasses bei gleichzeitig hoher Arbeitslosigkeit bestätigen.

Die Entwicklung auf dem deutschen Arbeitsmarkt verlief in den vergangenen Jahren durchaus positiv. Der Aufwärtstrend mit steigenden Beschäftigtenzahlen und sinkender Arbeitslosigkeit konnte auch durch die Finanz- und Wirtschaftskrise nicht umgekehrt werden. Diese guten Nachrichten werden jedoch getrübt von der Diskussion um einen zunehmenden Fachkräftemangel, der zu einer Schwächung der Leistungsfähigkeit der deutschen Betriebe und der Volkswirtschaft führen könnte. Zunächst ist es ein konjunkturelles Phänomen, dass die Personalrekrutierung in wirtschaftlich guten Zeiten schwieriger wird. Ein Blick auf Teilarbeitsmärkte zeigt, dass die Situation vor allem in pros- perierenden Regionen und bestimmten Berufssegmenten bereits angespannt ist. Diese Anzeichen enger werdender Arbeitsmärkte scheinen auch in der betrieblichen Wahrnehmung auf. Etwa jeder sechste Betrieb berichtet von einem Fachkräftemangel - westdeutsche Betriebe und größere Betriebe sind überdurchschnittlich oft betroffen. Dabei wird zwar auch bei der eigenen Belegschaft Verbesserungspotenzial gesehen, die meisten Betriebe beziehen sich aber auf Probleme im externen Rekrutierungsprozess. Es wird vor allem die zu geringe fachliche Eignung der Bewerber beklagt. Die Analyse von Neueinstellungen zeigt, dass besonders Betriebe in Westdeutschland bereit sind, im Wettbewerb um qualifizierte Mitarbeiter Lohnaufschläge anzubieten. Scheitern Rekrutierungsprozesse vollständig, so weisen die Betriebe eine gewisse Flexibilität auf, um die Personalprobleme aufzufangen. Nur in etwa jedem vierten Fall eines Suchabbruchs müssen Aufträge abgelehnt werden.

Künftig steht vor allem der Osten Deutschlands vor der Herausforderung, neben dem strukturellen Wandel auch auf einen rapiden demografischen Wandel zu reagieren. Zusätzlich zur gesamtdeutschen Verrentung geburtenstarker Nachkriegskohorten kommt es hier aufgrund der erst jüngst zurückgehenden hohen Nettobinnenabwanderungszahlen von nunmehr 2 Mio. jungen Menschen und einem transformationsbedingten Geburtenknick in Ostdeutschland zu einer harschen Schrumpfung und Alterung des Erwerbspersonenpotenzials in den nächsten Jahren, über dessen Geschwindigkeit in ostdeutschen Betrieben keine Erfahrungswerte vorliegen (Kubis et al. 2011). Auch in Westdeutschland ist die demografische Schrumpfung und Alterung der Gesellschaft nicht aufzuhalten, auch wenn die Wanderungsbewegungen aus dem Osten Deutschlands die demografisch bedingte Verknappung der Erwerbsbevölkerung gedämpft haben.

Derzeit sind in den prosperierenden Regionen im Süden Deutschlands die stärksten Anzeichen für Fachkräfteprobleme zu sehen. Zukünftig kann sich das Bild ändern und auch stärker den Osten Deutschlands betreffen. Denn dort stehen die Betriebe vor der Herausforderung, mit den wachsenden Wirtschaftszentren im Westen um die knapper werdende Ressource Fachkräfte zu konkurrieren: Ganz Deutschland befindet sich im Rahmen der anhaltenden Öffnung der Märkte in einem Wettbewerb um die besten Ideen und das beste Know-how. 


\section{LITERATUR}

Brenke, K. (2011): Droht Deutschland ein Fachkräftemangel? Contra, in: WirtschaftsWoche online vom 18.2., http://www.wiwo.de/archiv/pro-undcontra-droht-deutschland-ein-fachkraeftemangel/5740730.html (letzter Zugriff: 16.12.2012)

Dietz, M./Dittrich, L./Stops, M./Walwei, U. (2011): Beschäftigungssicherung durch Arbeitskräftehorten, in: Sozialer Fortschritt 60 (10), S. 221-231

Gartner, H./Klinger, S. (2007): Aufschwung am Arbeitsmarkt - Trendwende oder Strohfeuer?, in: Wirtschaftsdienst 87 (9), S. 613-619

Klinger, S./Rothe, T. (2012): The impact of labour market reforms and economic performance on the matching of the short-term and the long-term unemployed, in: Scottish Journal of Political Economy 59 (1), S. 90-114

Koppel, O. (2011): Ingenieure: Die Mär vom Schweinezyklus, Institut der deutschen Wirtschaft, IW-Nachrichten vom 07.08., Köln

Kubis, A./Lang, C./Schneider, L./ Heimpold, G. (2011): Ostdeutsche Transformationsgesellschaft: Zum Fortbestand von Strukturen und Verhaltensweisen, in: Lorenz, A. (Hrsg.): Ostdeutschland und die Sozialwissenschaften. Bilanz und Perspektiven 20 Jahre nach der Wiedervereinigung, Opladen, S. 189-207 Ragnitz, J./Ammon, M./Brenke K./Komar W./Pfankuch, J./Schneider, A./ Stierwald, A. (2004): Der Aufbau Ost als Gegenstand der Forschung. Untersuchungsergebnisse seit 1990. Projekt im Auftrag des Bundesamts für Bauwesen und Raumordnung (BBR), Bonn

\section{AUTOREN}

MARTIN DIETZ, Dr. rer. pol. (Volkswirtschaftslehre), Leiter der Forschungskoordination (kommissarisch) des Instituts für Arbeitsmarkt- und Berufsforschung (IAB), Nürnberg. Arbeitsschwerpunkte: Arbeitsmarktpolitik, Evaluationsforschung.

martin.dietz@iab.de

ALEXANDER KUBIS, Dr. rer. pol. (Volkswirtschaftslehre), Wissenschaftlicher Mitarbeiter im Forschungsbereich „Prognosen und Strukturanalysen" des IAB, Nürnberg. Arbeitsschwerpunkte: Fachkräftebedarf, Stellenbesetzungsprozesse, Ost-West-Migration.

alexander.kubis@iab.de

ANNE MÜLLER, Diplom- Sozialwirtin, Wissenschaftliche Mitarbeiterin im Forschungsbereich „Prognosen und Strukturanalysen" des IAB, Nürnberg. Arbeitsschwerpunkte: Fachkräftebedarf, Stellenbesetzungsprozesse, berufsspezifische Nachfrage.

anne.mueller@iab.de 